\title{
LA RAISON D'ETAT DANS LA ROME ANTIQUE: PROLÉGOMĖNES (CICÉRON, TITE-LIVE, TACITE)
}

\author{
Yasmina Benferhat*
}

\section{ABSTRACT}

This paper discusses the concept of raison d'Etat, or statecraft, in Rome under the Republic and the early Empire. The first part focuses on Republican times, when the Romans took extraordinary measures to stop a revolt abroad - Numance in $133 \mathrm{BC}$ and Corinth in $146 \mathrm{BC}$ - or to return order and stability to Rome with a senatusconsultum ultimum. This can be considered first steps where the State's survival was more important than the lives of citizens.

The second part deals with statecraft under the Roman Empire (first century $A D)$, considering the state as an entity independent from the prince. Examples deriving from Tacitus' Histories have been used to emphasise the fact that some Roman politicians took decisions in order to preserve the State, for example in 69 when Mucianus convinced Vespasian to rebel against two bad emperors, namely Otho and Vitellius.

* Maître de conférence habilitée, Université de Lorraine. 


\section{YASMINA BENFERHAT}

The third and last part deals with another point of view on the Roman Empire, namely that the raison d'Etat was actually the will of the emperor to save his own power and its dynastic transmission. It was often very difficult to distinguish between the Emperor and the State. The reigns of Tiberius and Nero offer a dark version of statecraft, full of dissimulation and violence against whoever was willing or able to reign after them. In consequence, the essay shows that the strong prejudice that Statecraft originated with Machiavelli and was unknown in ancient states is not correct and that the concept of raison d'Etat can be applied to ancient Rome.

Keywords: Statecraft; Rome; Cicero; Livy; Tacitus; politics; empire

"Omne magnum exemplum habet aliquid ex iniquo, quod contra singulos utilitate publica rependitur." Cet avis intervient dans le contexte bien connu de l'affaire Pedanius, un Préfet de la ville assassiné chez lui par certains de ses esclaves sous le règne de Néron. La coutume voulait qu'on exécutât dans ces cas-là tous les esclaves, ${ }^{2}$ complices ou non, qui se trouvaient dans la maison au moment des faits. Or certains s'émurent du grand nombre d'innocents qui allaient périr, mais Cassius ${ }^{3}$ plaida au Sénat pour le respect de la règle dans l'intérêt de l'Etat: le passage cité conclut son discours.

Cela correspond assez au sens que nous accordons au concept de raison d'Etat ${ }^{4}$ qui est d'abord le fait de mettre en avant l'intérêt de l'Etat aux dépens des particuliers, afin de justifier une action contraire à la justice. C'est placer l'intérêt de l'Etat audessus de la morale commune. Pour mémoire, le premier traité politique traitant la question de la "Ragione di stato" date de 1589. ${ }^{5}$ Mais avant Botero, auteur de ce traité, c'est Machiavel qui, sans utiliser l'expression de raison d'Etat, a le mieux exposé ses subtilités et ses nécessités. La raison d'Etat naîtrait donc à l'époque moderne quand l'Etat naît véritablement, bien après la Rome antique?

La République romaine n'avait assurément pas tous les attributs d'un Etat moderne - en particulier il lui manquait une force de police permanente ${ }^{6}$ qui assure l'ordre public et la sécurité - mais c'était un Etat solide avec un Sénat se chargeant de

1 Tacite Ann 14, 44, 4. Le discours de Cassius a fait l'objet d'une analyse de Nörr 1983: 187-222, qui traite rapidement des aspects juridiques pour analyser surtout les effets rhétoriques du discours et la présentation de Cassius comme chantre d'une seueritas peut-être obsolète à son époque.

2 Voir, concernant le contexte général du contrôle exercé sur les esclaves, Bradley 1987: 99-100, 131-134.

3 Sur ce sénateur, C Cassius Longinus, voir Liebs 2010: 25 (bibliographie dans la note 80).

4 De fait, Bellen a consacré une étude à cette affaire, intitulée "Antike Staatsräson", voir Bellen 1982: 449-467: c'est pourquoi nous avons choisi de commencer par cet exemple. Voir Meinecke 1998: 25-27 (il évoque Thucydide, Euripide chez les Grecs, Cicéron et Tacite pour Rome).

5 Nous laisserons de côté ici les prédécesseurs de Botero qui utilisèrent l'expression avant lui, comme Guicciardini et Della Casa: voir Bonnet 2003: 315-329.

6 Voir sur cette question Nippel 1995: 4-46, 120-121 pour un état de la question bibliographique. Lintott souligne bien le problème dans son ouvrage: voir Lintott 1999: 89 en particulier. 
la politique extérieure ainsi que des finances, une justice dotée de tribunaux devenus permanents et spécialisés depuis Sylla, enfin une armée efficace. Ses faiblesses étaient en partie compensées par une capacité indéniable - et parfois sous-estimée à savoir assouplir les cadres existants pour s'adapter à de nouvelles réalités. Dans un deuxième temps, Auguste a largement contribué à la mise en place d'un Etat pourvu de toutes les institutions permanentes permettant son bon fonctionnement ${ }^{7}$ et certains de ses successeurs, comme Claude qui a mis en place de véritables ministères, ont perfectionné la machine administrative impériale.

L'objet de notre étude sera de poser la question du rôle de Tacite dans la réflexion sur la raison d'Etat, en trois temps: nous verrons d'abord un premier sens de la raison d'Etat, à savoir la survie de l'institution aux dépens de l'individu prié de se sacrifier si besoin est, un sens bien présent à Rome dès la République. Dans un deuxième temps, nous verrons comment Tacite plus que d'autres eut le sens de l'Etat, sans lequel il n'y a pas de raison d'Etat. Enfin, nous étudierons dans un troisième temps la peinture des Julio-Claudiens dans les Annales, avec la confusion entre affaires publiques et affaires du Prince.

Une première façon d'envisager la raison d'Etat est de considérer que c'est la survie de l'institution passant par la subordination totale de l'individu à l'Etat. Ce phénomène peut être observé dès l'époque de la République: de fait, Tite-Live et avant lui Cicéron ont abordé la question. Les cas de figure peuvent être assez différents, mais ils se laissent néanmoins classer en deux grandes catégories. Considérons d'abord le dévouement d'un individu en temps de guerre: comment ne pas songer à Regulus ${ }^{8}$ dont l'histoire est connue en particulier par l'éloge de Cicéron ${ }^{9}$ dans le De finibus? Ce général romain, qui avait tenté un débarquement en Afrique lors de la première Guerre Punique et se retrouva aux mains des Carthaginois à la suite d'une défaite, ${ }^{10}$ fut envoyé comme ambassadeur à Rome avec pour mission de faire accepter à ses concitoyens un traité de paix: ${ }^{11}$ s'il échouait, les pires tortures lui étaient promises à son retour en Afrique. Or, Regulus plaida contre ce traité défavorable à Rome, et préféra donc le salut de l'Etat à la vie.

7 C'est ainsi qu'on trouve des institutions de police sous l'Empire: voir l'exemple de l'Asie mineure étudié par Brélaz 2005: 4-5 où il souligne l'absence de l'idée de police chez les Romains, en tant qu'organe administratif spécifiquement affecté au maintien de l'ordre, avant d'étudier les différentes solutions mises en place (pp 69-230 sur les institutions municipales, et pp 231-282 sur le rôle de l'armée pour garantir la sécurité publique).

8 Voir Mix 1970 qui synthétise toutes les données dont nous disposons sur Regulus et la mise en place de sa légende à Rome. Sur une comparaison entre Regulus et Duilius, autre général de la première Guerre Punique, voir Gendre \& Loutsch 2001: 136-163.

9 Cf Fin 2, 65. Cicéron cite à nouveau l'exemple de Regulus dans le De Officiis pour montrer qu'il choisit ce qui était utile à l'Etat au détriment de ce qui aurait pu lui être utile: cf Off 3, 99-100.

10 Cf Polybe 1, 30-35.

11 Il semble qu'il se soit agi d'un arrêt des combats, ou d'un échange de prisonniers. 
L'histoire connaît des variantes: les Periochae indiquent sobrement qu'il fut exécuté, d'autres sources insistent sur les tortures qui lui auraient été infligées. ${ }^{12}$ Cicéron met surtout en avant la fides de Regulus, le respect de la parole donnée au mépris de sa propre vie, dans sa présentation. Cependant, il serait possible de s'interroger sur les raisons du choix de Regulus, en se demandant si c'était un sens très élevé de l'intérêt de l'Etat qui lui était propre ou si cette façon de voir était partagée par tous, auquel cas on n'aurait pas ici, certes, une raison d'État fruit d'une autorité étatique imposant une décision, mais une forte pression du groupe poussant un individu à se sacrifier si nécessaire.

Un deuxième exemple est offert par le général romain vaincu aux Fourches Caudines. Postumius décide de se laisser livrer aux Samnites après son retour peu glorieux à Rome. ${ }^{13}$ Son sacrifice est alors loué par tous:

Postumius in ore erat; eum laudibus ad caelum ferebant, deuotioni P. Deci consulis, aliis claris facinoribus aequabant: emersisse ciuitatem ex obnoxia pace illius consilio et opera; ipsum se cruciatibus et hostium irae offerre piaculaque pro populo Romano dare. ${ }^{14}$

Postumius se sacrifie à la raison d'Etat bien plus que ne l'imaginent encore ses concitoyens: en effet, une fois amené aux Samnites pour leur être livré, il argue de sa nouvelle citoyenneté samnite, en tant que prisonnier, pour offrir un motif de bellum iustum à Rome en frappant un de ses gardes romains. C'était pousser très loin la logique de la raison d'Etat, puisque non seulement le consul permettait à sa patrie de ne pas se sentir engagée par un traité de paix désavantageux, mais au péril de sa vie il lui offrit même de quoi reprendre la guerre dans la meilleure des positions selon la logique romaine, celle de l'agressé. Dans des années très difficiles pour Rome, qui ne luttait pas seulement pour le contrôle de la Campanie mais quasiment pour son salut contre les Samnites alliés à d'autres peuples présents en Italie et désireux de prendre une revanche sur les Romains, la raison d'Etat est là sans qu'il soit besoin de la nommer. Cet Etat militaire, constamment en guerre depuis 509 avant Jésus-Christ ou presque, attend et obtient de ses citoyens un sacrifice personnel s'il en est besoin pour assurer le salut de la res publica.

On achèvera cette série d'exemples avec une famille célèbre pour avoir pratiqué régulièrement le rite de la deuotio: ${ }^{15}$ le premier fut le consul de 340 Publius Decius Mus au cours de la bataille de Véséris lors du soulèvement de la Ligue Latine. TiteLive décrit longuement ce rite et souligne à la fin que l'on aurait pu envoyer à la mort n'importe quel citoyen romain inscrit dans la légion, afin de mieux mettre en valeur le sacrifice de Decius. ${ }^{16}$ Son fils se dévoua à son tour à la bataille de

2 Cf Florus 2, 2 et Appien Pun 5 par exemple.

13 Cette deditio a été très bien analysée par Michel 1980: 679sqq.

14 Liv 9, 10, 3-4: voir le commentaire de Oakley 2005: 131-132 qui souligne que ce thème était annoncé déjà plus tôt, cf Liv 9, 4, 10 .

15 Sur ce rite et ses implications religieuses, voir Dumézil 1952.

$16 \operatorname{Liv} 8,9$ 
Sentinum ${ }^{17}$ en 295 , et son petit-fils fit de même à la bataille d'Asculum contre Pyrrhus en 279. On retrouve bien ici la survie de l'Etat assurée par le sacrifice d'individus, mais est-ce vraiment de la raison d'Etat au sens où nous l'entendons usuellement? N'est-ce pas plutôt un sens très élevé de l'intérêt de l'Etat qui amena des généraux à se sacrifier librement? Le Sénat n'exigeait pas d'eux expressément qu'ils meurent dans les conditions décrites plus haut: on peut évidemment penser qu'il devait y avoir une pression sociale énorme, mais il ne s'agit pas ici des innocents sacrifiés de façon injuste au nom de l'intérêt public.

En revanche, la destruction de Corinthe en 146 avant JC (tout comme la destruction de Carthage ou le martyre de Numance en Espagne) pourrait apparaître comme une application de la raison d'Etat puisque les Romains voulurent faire un exemple afin de marquer les esprits et de dissuader les peuples vaincus de se soulever à nouveau. La Grèce s'étant soulevée contre la domination romaine, Corinthe fut détruite et ses habitants massacrés ou vendus comme esclaves. Cicéron critique les Romains, et le cas l'embarrasse assez pour qu'il préfère glisser dessus en évoquant plus longuement un exemple grec:

Sed utilitatis specie in re publica saepissime peccatur, ut in Corinthi disturbatione nostri; durius etiam Athenienses qui sciuerunt ut Aeginetis qui classe ualebant, pollices praeciderentur. ${ }^{18}$

Le commentaire de Cicéron est sans appel: il condamne ces mesures extrêmes comme de la cruauté totalement inutile avec les termes species - il s'agit d'une illusion d'intérêt pour l'Etat - et peccare surtout qui signale une erreur. C'est que dans un ouvrage qui tient beaucoup du testament philosophique - mais surtout politique comme très souvent chez Cicéron - l'opposition de principe entre l'utile et le beau, entre ce qui rend service et ce qui est conforme à la justice, qui est à la base de la raison d'Etat en fait, est clairement refusée. ${ }^{19}$ Le beau (autrement dit, ce qui est juste) et l'utile sont inséparables, et ne forment qu'un du point de vue de Cicéron. ${ }^{20}$

Cette réflexion est - à notre sens - révélatrice de l'absence de consensus au sein des élites romaines à propos de ces mesures exceptionnelles: plus que de raison d'Etat il faudrait souvent parler de raison d'un individu qui réussit à convaincre ses pairs au Sénat, l'exemple le plus célèbre étant Caton l'Ancien dont l'obstination l'emporta, alors que Scipion Nasica était beaucoup moins convaincu de la nécessité de détruire l'ancienne rivale Carthage. ${ }^{21}$ Plus largement, elle montre aussi les limites de l'utilisation possible de la raison d'Etat dans un régime qui n'est pas dirigé par une

17 Cf Liv 10, 27-29, 20: voir Oakley 2005: 275-277 (analyse générale soulignant les différences dans la présentation de la devotio du père et celle du fils) et 313-329 (commentaire du texte).

18 Cicéron Off 3, 11, 46.

19 Cf Cicéron Off 1,159 et 3, 20-35 en particulier.

20 Sur la fausse opposition entre beau et utile, cf Off 1, 9-10: c'est néanmoins l'enjeu du livre 3 du De Officiis, surtout, cf Off 3, 7sqq. Voir Nemo 1998: 340.

21 Sur l'opposition entre Caton le Censeur et Scipion Nasica au sujet de Carthage, voir Gelzer 1963: 39-72. 
seule personne - tout entourée qu'elle soit de ses conseillers et ministres - pouvant imposer sa vision de l'intérêt public à tous.

Qu'en était-il en cas de troubles politiques intérieurs? Il faut se pencher sur l'utilisation du senatusconsulte ultimum $^{22}$ adopté en 121. La formulation de cette proclamation de l'état d'urgence, pour résumer l'enjeu, est connue grâce à Cicéron qui la cite dans la Première Catilinaire: "Decreuit quondam senatus uti L. Opimius consul uideret ne quid res publica detrimenti caperet." ${ }^{23}$

Le principe était que les institutions régulières seraient suspendues le temps de mettre fin au désordre, et surtout cette mesure exceptionnelle permettait d'exécuter des individus jugés responsables d'une agitation dangereuse pour l'Etat, ainsi que leurs complices, sans avoir à passer par l'assemblée populaire et sans tenir compte de leur rang ou de leur magistrature. Cicéron décrit très bien cela, toujours dans la Première Catilinaire:

nox nulla intercessit; interfectus est propter quasdam seditionum suspiciones C. Gracchus, clarissimo patre, auo, maioribus, occisus est cum liberis M. Fuluius consularis. Simili senatus consulto C. Mario et L. Valerio consulibus est permissa res publica; num unum diem postea L. Saturninum tribunum pl. et C. Seruilium praetorem mors ac rei publicae poena remorata est? ${ }^{24}$

Le consul de 63 souligne ici premièrement la rapidité d'action - on ne remit pas au lendemain - puis la sévérité, puisqu'il n'y avait que des soupçons de sédition, et enfin la place au sein de l'Etat tenue par les victimes de la répression: Caius Gracchus avait été tribun de la plèbe, Fulvius était ancien consul.

Cicéron rappelle également que le senatusconsulte ultimum fut plusieurs fois utilisé contre des tribuns de la plèbe jugés séditieux, et donc dangereux pour l'Etat, puisque le Sénat proclama l'état d'urgence à nouveau contre Saturninus et son complice Servilius coupables d'avoir organisé l'assassinat en plein Forum d'un concurrent et responsables des troubles qui suivirent. ${ }^{25}$ Cela se passa en 100 avant JC sous le sixième consulat de Marius qui fut bien obligé de sévir contre ceux qui étaient alors ses alliés politiques, la raison d'Etat l'emportant sur ses propres intérêts.

On objectera que le senatusconsulte ultimum n'était qu'une arme forgée par une partie de l'Etat - les sénateurs - contre une autre partie de l'Etat - les tribuns de la plèbe - et que l'on ne saurait parler de raison d'Etat dans ces conditions. C'est tout l'enjeu du Pro Rabirio, un discours prononcé par Cicéron en 63 pour la défense d'un chevalier romain âgé, Rabirius, accusé d'avoir participé à l'exécution du tribun Saturninus ... ou plutôt à son meurtre, aurait dit son accusateur, Labienus, qui faisait

25 Voir Doblhofer 1990 sur les Populares des années 111-99 et plus particulièrement at 73-88 sur Saturninus dont une biographie a été écrite par Cavaggioni 1998: 144-157 sur le senatus consulte ultimum utilisé contre ce tribun. 


\section{LA RAISON D'ETAT DANS LA ROME ANTIQUE}

partie des Populares: ces enfants spirituels des Gracques contestaient la validité du senatusconsulte ultimum qui selon eux bridait la population, portait atteinte à la sacrosanctitas des représentants de la plèbe et remettait en cause la souveraineté populaire. La polémique est résumée par Cicéron au début de son discours:

Non enim C. Rabirium culpa delicti, non inuidia uitae, Quirites, non denique ueteres iustae grauesque inimicitiae ciuium in discrimen capitis uocauerunt, sed ut illud summum auxilium maiestatis atque imperi quod nobis a maioribus est traditum de re publica tolleretur, ut nihil posthac auctoritas senatus, nihil consulare imperium, nihil consensio bonorum contra pestem ac perniciem ciuitatis ualeret, idcirco in his rebus euertendis unius hominis senectus, infirmitas solitudoque temptata est. ${ }^{26}$

On notera d'abord que le senatusconsulte ultimum est décrit dès le début comme un moyen de porter secours à l'Etat. Surtout Cicéron insiste sur toutes les forces en présence qui sont protégées par le senatusconsulte ultimum:

Quam ob rem si est boni consulis, cum cuncta auxilia rei publicae labefactari conuellique uideat, ferre opem patriae, succurrere saluti fortunisque communibus, implorare ciuium fidem, suam salutem posteriorem salute communi ducere, est etiam bonorum et fortium ciuium, quales uos omnibus rei publicae temporibus exstitistis, intercludere omnis seditionum uias, munire praesidia rei publicae, summum in consulibus imperium, summum in senatu consilium putare; ea qui secutus sit, laude potius et honore quam poena et supplicio dignum iudicare. $^{27}$

Certes il y a les sénateurs, mais il y a également l'autorité des consuls, mais il y a tous les boni - les bons citoyens - d'accord pour lutter contre un fléau qui menace l'Etat. Ensuite, les consuls chargés d'appliquer l'état d'urgence doivent mobiliser la population en étant prêts à se sacrifier eux-mêmes pour le salut de l'Etat: si ce n'est pas de la raison d'Etat ... Enfin, tous les citoyens dignes de ce nom doivent prêter mainforte contre les fauteurs de trouble. Cicéron décrit longuement la répression mise en place contre Saturninus et Servilius afin de mettre en lumière ce consensus: ce fut bien une large majorité de la population qui aida à l'application du senatusconsulte ultimum, donc ce fut bien l'Etat qui se défendit contre quelques citoyens pernicieux.

Si l'on ne regarde que le résultat, l'application du senatusconsulte ultimum tient de la mise en place d'une raison d'Etat: on sacrifie des citoyens, des particuliers, au bien commun - l'utilitas publica, en les exécutant afin d'assurer le bon fonctionnement des affaires publiques. Mais du point de vue de Cicéron, ${ }^{28}$ du point de vue de ceux qui votèrent cette proclamation de l'état d'urgence, c'était une autre affaire, parce qu'ils considéraient que les victimes de la répression étaient bien coupables: il n'y

26 Cic Rab 1, 2.

27 Cic Rab 1, 3.

28 Cicéron condamne dans le De Officiis toute gouvernance s'appuyant sur la cruauté et la crainte, autrement dit une certaine idée de l'utile pour le gouvernant aux dépens de ses administrés, cf $O f f$ 2,23 sqq. 
avait point d'injustice dans ces conditions, point de sacrifice d'innocents au nom de la survie de l'Etat, mais une nécessaire réaction contre des citoyens séditieux.

Cette présentation du senatusconsulte ultimum ne correspond pas à la façon dont Tacite lui-même voyait les choses ... Il décrit la fin de la République comme un temps où l'Etat tangue, déchiré par les divisions et les ambitions:

Vetus ac iam pridem insita mortalibus potentiae cupido cum imperii magnitudine adoleuit erupitque; nam rebus modicis aequalitas facile habebatur. sed ubi subacto orbe et aemulis urbibus regibusue excisis securas opes concupiscere uacuum fuit, prima inter patres plebemque certamina exarsere. Modo turbulenti tribuni, modo consules praeualidi, et in urbe ac foro temptamenta ciuilium bellorum; mox e plebe infima C. Marius et nobilium saeuissimus L. Sulla uictam armis libertatem in dominationem uerterunt. ${ }^{29}$

Cette présentation doit beaucoup à Salluste, Tacite s'étant ici inspiré de la digression sur les guerres civiles présente dans la Guerre de Jugurtha. ${ }^{30}$ Elle est assez surprenante à première vue de la part d'un haut fonctionnaire avec un sens de l'Etat très élevé qui écrit plus d'un siècle après. Sans revenir sur les détails de la carrière de l'historien et les querelles d'érudits sur le fait de savoir si son père était le fameux procurateur de Belgique Cornelius Tacitus ou non, ${ }^{31}$ il convient de retenir que par son parcours et ses relations il appartenait au milieu des grands commis de l'Etat: son épouse était la fille d'Agricola, consul en 77 et gouverneur de Bretagne. Pline le Jeune son ami était également haut fonctionnaire. Les deux ont connu plusieurs crises politiques de la fin du règne de Néron jusqu'à l'avènement de Trajan et les deux ont choisi de faire fonctionner le système, même sous un mauvais Prince. Tacite critique d'ailleurs une opposition stérile qui aboutit à l'élimination d'hommes de valeur qui auraient pu servir l'Etat. ${ }^{32}$

De ce fait, la raison d'Etat est bien présente chez Tacite, mais il faut préciser que l'Etat est une entité indépendante de la succession des Princes et donc des empereurs eux-mêmes. C'est d'ailleurs une première clé pour comprendre la critique des dernières années de la République quand des individus essaient de s'emparer du pouvoir en nuisant au bon fonctionnement des institutions. La raison d'Etat que l'on peut repérer dans les Histoires en particulier, du fait de la dissociation forcée de l'Etat et des Princes trop éphémères pour ne faire qu'un avec lui, est un concept de haut fonctionnaire: elle aurait pu intéresser un Richelieu, grand serviteur de l'Etat ... Elle

29 Tacite Hist 2, 38: voir le commentaire de Syme 1982: 142-144. L'expression e plebe infima est assez curieuse pour Marius, mais c'est probablement pour créer un contraste avec Sylla et grossir même artificiellement les différences sociales qui existaient entre les deux ennemis.

30 Cf Salluste $B J 41-42$. Il est à relever que la narration de la Conjuration de Catilina ne laisse pas deviner d'aversion particulière au senatusconsulte ultimum, $\operatorname{cf} C C 29,3$ alors que César y était nettement moins favorable.

31 Nous renvoyons à Syme 1958: 59-75.

32 Cf Tacite Agric 42, 6 et Ann 16, 16, 1. 
a un côté très moderne qui n'est pas sans faire songer à la façon dont la Quatrième République a pu exister en France pendant douze ans malgré l'instabilité chronique des gouvernements, parce que la machine fonctionnait grâce à l'administration.

Le premier exemple de présence de la raison d'Etat, le plus évident d'ailleurs, c'est le choix d'un Prince. Cela se voit dans le discours de Galba à Pison: ${ }^{33}$ dans ce passage qui doit sans doute beaucoup à une adoption beaucoup plus récente du point de vue de Tacite - celle de Trajan par Nerva en 98 - le vieil empereur énumère les raisons de son choix. Il doit à l'Etat un successeur désigné jeune pour éviter l'instabilité: il va le choisir dans une des familles les plus nobles de Rome, ce qui assure là encore une continuité. Et il s'attache à un homme qui aime sa patrie, en plus d'avoir les qualités d'un bon dirigeant. La raison d'Etat est là quand Galba fait le procès des mauvais conseillers qui préfèrent abonder dans le sens du Prince plutôt que lui dire ce qu'il doit faire: "Nam suadere principi quod oporteat multi laboris, adsentatio erga quemcumque principem sine adfectu peragitur." ${ }^{34}$

Elle est là aussi dans le choix du meilleur, ce qui passe par le sacrifice de ses propres enfants qui n'ont pas les mêmes qualités. Ce problème du choix d'un Prince permettant la survie de l'Etat est en réalité présent dans l'ensemble des deux premiers livres des Histoires, quand les hauts-fonctionnaires se désespèrent de devoir choisir entre un Othon et un Vitellius, jugés l'un comme l'autre pernicieux et dangereux pour Rome. Et finalement l'on pourrait voir en la candidature de Vespasien une réaction dictée par la raison d'Etat à l'œuvre afin de garantir à Rome un Prince capable d'assurer le bon fonctionnement des institutions: $:^{35}$ quoi que l'on puisse penser de Mucien, et Tacite ne l'embellit certes pas, le discours qu'il tient à Vespasien pour le convaincre d'être candidat à l'Empire est marqué du sceau de la raison d'Etat. Il s'agit d'être utile à l'Etat - rei publicae utile-, de le sauver même, comme le montre l'adjectif salutare également employé par Mucien qui précise juste après sa pensée:

non aduersus diui Augusti acerrimam mentem nec aduersus cautissimam Tiberii senectutem, ne contra Gai quidem aut Claudii uel Neronis fundatam longo imperio domum exurgimus; cessisti etiam Galbae imaginibus: torpere ultra et polluendam perdendamque rem publicam relinquere sopor et ignauia uideretur ... ${ }^{36}$

Vespasien se doit à l'Etat menacé de ruine par Vitellius; ce n'est qu'ensuite que Mucien use d'un autre argument, celui de la sécurité personnelle de Vespasien. On pourrait arguer qu'il s'agit de rhétorique fallacieuse pour pousser Vespasien au crime: on peut cependant considérer que Mucien, lui aussi haut-fonctionnaire, commence par des motifs nobles, les plus susceptibles de toucher un autre haut-fonctionnaire comme Vespasien. ${ }^{37}$

33 Tacite Hist 1, 15-16.

34 Tacite Hist 1, 15, 4 .

35 Sur les concurrents de la guerre civile de 69-70, voir Morgan 2006, et plus ancien Wellesley 2000.

36 Hist 2, 76.

37 Sur Vespasien et Mucien, voir Levick 1999 (chapitre 4 en particulier). 
Paradoxalement, c'est un des deux mauvais Princes cités comme repoussoirs par Mucien qui agit selon la raison d'Etat au crépuscule de sa vie, en décidant de se suicider plutôt que de relancer la guerre contre Vitellius en causant la ruine de milliers d'hommes et la destruction de l'Italie: "an ego tantum Romanae pubis, tot egregios exercitus sterni rursus et rei publicae eripi patiar? eat hic mecum animus, tamquam perituri pro me fueritis, set este superstites." 38

Othon emploie également l'expression remisisse rei publicae nouissimum casum: il a épargné à l'Etat un nouveau malheur alors que ses soldats étaient prêts à livrer bataille. ${ }^{39}$ Et il va veiller à lui en éviter d'autres par ses dernières mesures, en particulier la destruction de lettres compromettantes qui auraient été l'occasion de représailles: "libellos epistulasque studio erga se aut in Vitellium contumeliis insignis abolet; pecunias distribuit parce nec ut periturus." ${ }^{40}$ Libre à Mucien, comme à Tacite d'ailleurs, de considérer que ce ne fut pas la raison d'Etat mais le désespoir et l'incapacité à supporter l'attente qui amenèrent Othon à arrêter les combats. Libre aux lecteurs des Histoires de songer que le souci bien humain de la gloire et de la postérité fut aussi un motif important du choix d'Othon, d'autant qu'il gomme soigneusement sa propre responsabilité dans le meurtre de Galba pour accuser Vitellius d'être responsable de la guerre civile. Il n'empêche que la raison d'Etat est bien présente, en filigrane, en référence.

On donnera un dernier exemple qui concerne cette fois la gestion de l'Empire: c'est le discours de Petilius Cerialis, au livre IV des Histoires, lorsqu'il justifie l'impérialisme romain devant les Trévires et les Lingons. ${ }^{41}$ Un premier point essentiel est la conscience d'une continuité de l'Etat: "octingentorum annorum fortuna disciplinaque compages haec coaluit, quae conuelli sine exitio conuellentium non potest ..." ${ }^{42}$ Le deuxième point à retenir est l'application de la raison d'Etat à la gestion de l'Empire, et sa description très réaliste. Il faut des armées pour garantir la paix; ces armées doivent être payées; donc il faut des impôts:

Nos, quamquam totiens lacessiti, iure uictoriae id solum uobis addidimus, quo pacem tueremur; nam neque quies gentium sine armis neque arma sine stipendiis neque stipendia sine tributis haberi queunt: cetera in communi sita sunt. ${ }^{43}$

Les Gaulois doivent supporter ces tributs pour permettre à l'Etat de fonctionner, à leur avantage puisqu'il leur offre la paix et une intégration, certes progressive mais réelle. C'est la raison d'Etat qui veut que les Romains garantissent leurs frontières en intervenant en Gaule.

Hist 2, 47.

39 Sur ce passage voir le commentaire de Ash 2007: 203-207.

40 Hist 2, 48.

41 Voir le commentaire de Chilver \& Townend 1985: 78-80 et Ternes 1990: 112-122.

42 Hist $4,74$.

43 Ibid. 
Le troisième point retiendra particulièrement notre attention: c'est la distinction nette de 1'Etat et des Princes. ${ }^{44}$ Vita breuis, res publica longa ... Cerialis montre clairement que l'institution fonctionne grâce à des rouages anciens et solides, que les empereurs soient bons ou mauvais puisque de toute façon leur règne a une fin:

\begin{abstract}
et laudatorum principum usus ex aequo quamuis procul agentibus: saeui proximis ingruunt. quo modo sterilitatem aut nimios imbris et cetera naturae mala, ita luxum uel auaritiam dominantium tolerate. uitia erunt, donec homines, sed neque haec continua et meliorum interuentu pensantur. ${ }^{45}$
\end{abstract}

Un Tacite, un Agricola, un Pline auraient pu tenir semblables propos: on a ici une conception de la raison d'Etat vue par un haut fonctionnaire soucieux du bon fonctionnement de la machine et qui y participe de son mieux. De ce point de vue, Tacite n'est pas l'inventeur de la raison d'Etat, mais il est sans doute son meilleur metteur en scène par son talent d'historien et d'écrivain plus largement.

Néanmoins, il y a une autre façon de voir qui perce dans la description du règne des Julio-Claudiens, dans les Annales, en particulier. Lors de ces années de mise en place d'un régime monarchique, la personne du Prince était indissociable de l'Etat sur la lancée de ce qu'Auguste put représenter aux yeux de ses contemporains. Certes, en théorie il y avait distinction par exemple entre la fortune de l'Empereur et le Trésor public: Auguste insiste sur ce qui provient de son patrimoine personnel avec les expressions ex patrimonio meo, ${ }^{46}$ pecunia mea ${ }^{47}$ ex horreo et patrimonio meo, ${ }^{48}$ afin de mettre en valeur sa générosité envers ses concitoyens. Mais les excès d'un Caligula $^{49}$ montrent bien les limites du système.

Néron s'efforça pendant un certain temps de marquer la différence, à la manière d'un Auguste, entre son propre patrimoine et le trésor public: il souligne qu'il aide l'Etat de soixante millions de sesterces par an ${ }^{50}$ il aide avec ses propres revenus sua pecunia ${ }^{51}$ - les victimes de l'incendie de Rome. Néanmoins, ses folles dépenses en constructions et les récompenses versées à ses favoris l'amenèrent lui aussi à dilapider patrimoine des Césars et ressources de l'Etat ensemble: il eut donc recours aux mêmes expédients que Caligula à la fin de son règne..$^{52}$

44 Sur cette question voir Fritz 1957: 79-81: l'auteur souligne, entre autres, que les provinces se trouvèrent mieux sous les "mauvais" empereurs que sous le règne des "bons" Princes.

45 Ibid.

46 Cf Auguste $R G 15,1$.

47 Idem 17, 1: "Quater pecunia mea aerarium iuui ..."

48 Idem 18.

49 Cf Suétone Cal 37-42.

50 Cf Tacite Ann 15, 18, 3.

51 Idem 15, 43, 2.

52 Cf Suétone Ner 30-32. 
De même, il arriva que la justice fut rendue dans la domus du Prince sur le Palatin, sous Claude: ${ }^{53}$ la séparation entre justice d'Etat et arbitraire de l'Empereur était alors bien théorique ... On retiendra comme principal exemple l'affaire Valerius Asiaticus, victime de la jalousie de l'impératrice Messaline, et "jugé" dans les appartements de l'empereur en lieu et place du Sénat: "Neque data senatus copia; intra cubiculum auditur, Messalina coram et Suillio corruptionem militum, quos pecunia et stupro in omne flagitium obstrictos arguebat." ${ }^{54}$ Les scandales s'étant accumulés, une des premières mesures promises par Néron au début de son règne fut d'éviter le mélange des genres: discretam domum et rem publicam.$^{55} \mathrm{Le}$ même Claude contribua certainement à une plus grande efficacité de la machine en créant en quelque sorte des ministères, mais comme il mit à leur tête ses propres affranchis, l'Etat risquait bien d'apparaitre comme placé entre les mains du Prince. On peut ajouter à cela que même dans les provinces le Prince avait ses hommes, le procurateur en particulier, pour surveiller les hommes du Sénat, ce qui aboutissait en quelque sorte à deux administrations parallèles et concurrentes, sachant que le dernier mot revenait à l'Empereur.

Par conséquent, il est vrai qu'avec le régime mis en place sous les JulioClaudiens l'on trouve dans le récit de Tacite une raison d'Etat qui est en fait la raison du Prince, on aurait envie de dire le conatus regnandi d'un individu. Elle se caractérise par plusieurs traits: l'élimination des concurrents, réels et potentiels, le secret et l'opacité, la concentration du pouvoir sur la personne du Prince et son entourage avec de ce fait le difficile problème des favoris. La confusion opérée entre intérêt du Prince - la survie de son pouvoir - et intérêt de l'Etat est particulièrement redoutable pour le bon fonctionnement des affaires publiques, surtout à Rome.

Toutes ces facettes de la raison d'Etat à l'oeuvre dans les Annales's s'entremêlent en effet quand il s'agit en particulier de l'exécution des capaces imperii, pour reprendre la formule de Tacite, ${ }^{56}$ ces hommes qui se posaient en rivaux ou pouvaient apparaître comme tels, c'est-à-dire des sénateurs membres de grandes familles et ou avec une carrière prestigieuse. C'est sans doute le processus d'élimination des concurrents de Néron qui est le plus visible et le plus connu. Les premiers dangers immédiats pour le règne de Néron - à savoir les deux enfants de Claude - furent écartés par Agrippine: Octavie fut mariée à Néron tandis que Britannicus était marginalisé. Les meurtres commencèrent avec l'empoisonnement de Claude qui menaçait de remettre en cause les plans de la mère de Néron, puis la mort de Britannicus. On passa à une autre échelle quelques années plus tard, lorsque précisément le quinquennium, ces cinq premières années de règne jugées bénéfiques, prit fin.

Commença alors une période où Néron considéra comme raison d'Etat ce qui correspondait à ses propres vœux et inquiétudes. Pour reprendre rapidement des

53 Sur ce Prince voir Levick 1993. Sur sa façon de rendre la justice, voir Tuori 2016: 156.

54 Tacite Ann 11, 2, 1.

55 Tacite Ann 13, 4, 2.

56 Il l'emploie pour Galba en premier, cf Hist 1, 49, 4. 
événements bien connus de tous, Néron voulait répudier Octavie pour épouser Poppée, mais Agrippine s'y opposait. Assurément les sentiments des uns et des autres jouèrent un rôle, mais du point de vue de Néron il y avait probablement d'un côté le passé - Octavie qui était indispensable pour lui donner de la légitimité au moment de son accession au trône - et de l'autre l'avenir représenté par Poppée. Or un Prince a besoin d'un héritier et Octavie ne semblait pas pouvoir le lui donner. Donc la raison d'Etat selon Néron consistait à choisir l'avenir, en considérant qu'il était déjà Empereur depuis plusieurs années et qu'il n'avait plus besoin de sa première femme.

Agrippine fut éliminée, d'une part parce qu'elle s'opposait au projet d'union avec Poppée ${ }^{57}$ d'autre part parce qu'elle représentait désormais un facteur de troubles incessants. En effet, mécontente de son fils elle menaçait de lui susciter des concurrents depuis un moment: ${ }^{58}$ Britannicus ou Cornelius Sulla ou Plautus ... Or elle avait une certaine popularité, en particulier auprès de l'armée et des prétoriens, en tant que fille de Germanicus. Il est difficile de comprendre pourquoi Sénèque et Burrus se sont rangés du côté de Néron:

Igitur longum utriusque silentium, ne inriti dissuaderent, an eo descensum credebant ut, nisi praeueniretur Agrippina, pereundum Neroni esset. Post Seneca hactenus promptius <ut $>$ respiceret Burrum ac sciscitaretur an militi imperanda caedes esset. ${ }^{59}$

Les deux hommes de confiance du Prince, choisis par Agrippine, semblent hésiter devant l'annonce de l'échec d'une première tentative de matricide; puis Sénèque tire la conclusion qui semble s'imposer. Il est devenu trop dangereux de laisser Agrippine en vie, puisqu'elle sait que son fils a tenté de l'éliminer et qu'il s'agira désormais d'une lutte à mort entre eux deux. On retrouve ici le secret, le conseil des proches du pouvoir et la décision de procéder à un acte que la morale réprouve. Quant à Octavie, en tant que fille de Claude elle représentait, qu'elle le veuille ou non, une source d'insécurité pour le pouvoir de Néron: celui-ci se décida à la faire exécuter après des manifestations populaires en faveur de la jeune femme. ${ }^{60}$

A partir du moment où Néron était arrivé au trône dans des circonstances qui n'étaient pas celles - indiscutables - de la succession d'un fils légitime à son père, il ne pouvait qu'être pris dans un cercle vicieux d'éliminations successives, d'autant que la politique de mariages organisés par Auguste multipliait le nombre de capaces imperii qui avaient autant de titres à prétendre au trône que le rejeton d'Agrippine. ${ }^{61}$ L'objectif se mêle au subjectif, les motifs réels de crainte aux frayeurs du Prince: ce

57 Cf Tacite Ann 14, 1, 1-2.

58 Après la mort de Britannicus, Agrippine avait mis en avant Rubellius Plautus qui descendait lui aussi d'Auguste: cf Ann 13, 19-20. Agrippine fut menacée (idem 13, 21), tandis que Rubellius Plautus fut éliminé peu après.

59 Ann 14, 7, 3

60 Cf Ann 14, 61-64.

61 Voir Syme: 1989 (on pourra en particulier se référer aux arbres généalogiques qui se trouvent à la fin de cet ouvrage). 
que l'on constate, c'est que Tacite était sans doute bien conscient de cette situation, mais il a complètement masqué cette complexité dynastique pour présenter Néron comme un monstre. ${ }^{62}$

Néanmoins, les six premiers livres des Annales semblent bien offrir le plus de matière à réflexion sur la raison d'Etat, avec la figure de Tibère. Son règne fut long, qui dura vingt-trois ans, et s'acheva par une mort dans le calme; ce fut une période sans guerre ruineuse ou calamiteuse, parce que ce Prince privilégia la diplomatie. Et de ce fait, les caisses de l'Etat étaient bien remplies à sa mort. Tout cela fut obtenu par une certaine politique et par la conduite d'un homme à la tête de l'Etat. La qualité principale reconnue à Tibère par les penseurs politiques fut sa capacité de dissimulation: c'est en effet une des clés de la raison d'Etat. De fait Tacite le décrit comme renfermé et enclin à cacher les mauvaises nouvelles. ${ }^{63}$ Surtout il demeure mystérieux sur ses propres intentions et ne laisse pas deviner aisément ce qu'il va faire, d'autant qu'il n'hésite pas à utiliser des leurres comme ces préparatifs faits au moment de la sédition en Germanie pour faire croire qu'il allait s'y rendre afin de ramener le calme. ${ }^{64}$

On pourrait, néanmoins, considérer que Tibère était surtout quelqu'un qui n'aimait pas s'exprimer. Le véritable théoricien de la raison d'Etat est Sallustius Crispus qui énonce ses règles au début des Annales, lorsqu'il fallut gérer l'exécution du jeune Agrippa Postumus: ${ }^{65}$

quod postquam Sallustius Crispus particeps secretorum (is ad tribunum miserat codicillos) comperit, metuens ne reus subderetur, iuxta periculoso ficta seu uera promeret, monuit Liuiam ne arcana domus, ne consilia amicorum, ministeria militum uulgarentur, neue Tiberius uim principatus resolueret cuncta ad senatum uocando: eam condicionem esse imperandi, ut non aliter ratio constet quam si uni reddatur. ${ }^{66}$

L'assassinat du petit-fils d'Auguste, qu'il ait été commandé par celui-ci dans son testament ou fomenté par Livie et Tibère, n'allait pas manquer d'être critiqué: Tibère choisit donc de se décharger de toute responsabilité en annonçant que la chose serait discutée au Sénat. Crispus rappelle alors le Prince à l'ordre par l'intermédiaire de Livie: certaines affaires demandent le secret et ne doivent être sues que par l'Empereur. En réalité, Sallustius Crispus cherche surtout à se protéger: on voit ici, en plus du culte du secret qui permet d'éliminer discrètement des concurrents, l'importance des entourages qui sont là pour conseiller le Prince et le protéger, mais ont également à cœur leurs propres intérêts, souvent. Tibère connut ainsi une crise importante avec

62 Il est de ce fait assez paradoxal de voir Tacite utilisé pour trouver des recettes de politique au service de la raison d'Etat.

63 Cf Ann 1, 24.

64 Cf Ann 1, 47.

65 Sur cet épisode, voir l'analyse de Fritz: 1957 at 81-83: il conclut que l'exécution fut bien ordonnée par Auguste lui-même, ce qui nous semble le plus probable.

66 Ann 1, 6. 
la conjuration de Séjan, son trop ambitieux préfet du prétoire qui se voyait bien lui succéder, mais il sut s'en débarrasser à temps au moyen d'une répression féroce aux dépens de nombreux innocents comme les propres enfants de Séjan. ${ }^{67}$

Celui-ci avait intrigué pendant des années ${ }^{68}$ à la cour en séduisant d'abord la bru de Tibère pour la convaincre d'empoisonner Drusus, son mari et le fils du Prince. Puis, une fois ce premier héritier naturel mort, il avait sévi au détriment de la famille de Germanicus: sa veuve Agrippine et leurs nombreux enfants. Tous les garçons furent victimes de ses intrigues, à l'exception de Caligula, puisqu'ils représentaient une alternative. Mais les amis et soutiens d'Agrippine ne furent pas plus épargnés: Séjan lança contre eux des accusateurs, afin d'isoler et d'affaiblir le parti adverse. On a donc la description d'une cour en proie à la division du fait de la question lancinante de la succession du Prince, compliquée par les choix d'Auguste qui avait imposé à Tibère d'adopter son neveu Germanicus alors qu'il avait un fils.

Comment ce processus d'élimination fut-il mis en place? Tibère avait remis d'actualité en 21 la lex de maiestate ${ }^{69}$ qui permit d'abattre un bon nombre de personnes selon les intrigues du moment. Ce fut l'arme de la raison d'Etat: cette loi, qui datait des temps républicains, permettait à l'origine de punir les trahisons contre la patrie, mais aussi à l'occasion des affronts faits au peuple romain. On pensera à la célèbre vestale Claudia trouvant qu'il y avait décidément trop de monde sur le Forum pour circuler tranquillement et souhaitant à haute voix une défaite navale de plus avec beaucoup de morts - c'était l'époque des Guerres Puniques - pour que la foule soit moins importante.

Si Auguste avait évité de s'en servir, Tibère la remit en vigueur à son propre bénéfice: ce n'était plus la maiestas du peuple romain qui était en jeu, mais celle du Prince. Et le champ d'application devint infini ou presque: à côté des tentatives de complot, la lex de maiestate pouvait toucher des personnes qui avaient simplement émis des regrets de voir Agrippine maltraitée, des personnes qui auraient porté atteinte à la dignité du Prince à leur domicile en ne montrant pas assez de respect pour une statue ou un portrait sur une bague. Cette loi encourageait le développement de la délation: il pouvait y avoir des motifs personnels, mais certains n'eurent aucun scrupule à servir de vecteurs des mauvaises intentions de Tibère (ou de Séjan quand il était en grâce). La maiestas du Prince permit ainsi de se débarrasser de beaucoup de gens de manière très commode grâce à un concept flou.

\section{Conclusion}

Nous avons pu constater que la raison d'Etat, loin d'être un anachronisme, existait déjà dans la Rome antique: c'est l'utilitas publica, ce qui rend service à l'Etat aux 
dépens parfois des particuliers. Les historiens romains ont été particulièrement attentifs à ce concept, qu'il s'agisse de Tite-Live ou de Tacite, sans oublier Salluste, parce qu'un de leurs objectifs était d'offrir à leurs lecteurs des leçons pour une bonne gestion de l'Etat et de l'imperium Romanum. Historia magistra ... Et parce que l'histoire à Rome n'est pas seulement enseignement, mais également divertissement, en puisant à d'autres genres littéraires, en s'inspirant d'autres procédés stylistiques, les historiens romains et peut-être en particulier Tacite ont su marquer les esprits par leur peinture saisissante du fonctionnement d'un Etat. Belle leçon qui nous est parvenue à travers les siècles ...

\section{BIBLIOGRAPHY}

Ash, R (2007) Tacitus. Histories Book II (Cambridge)

Bauman, R (1967) The Crimen Maiestatis in the Roman Republic and Augustan Principate (Johannesburg)

Bellen, H (1982) “Antike Staatsräson. Die Hinrichtung der 400 Sklaven des römischen Stadtpräfekten L. Pedanius Secundus im Jahre 61 n. Chr.” Gymnasium 89: 449-467

Bonnet, S (2003) "Botero machiavélien ou l'invention de la raison d'Etat" Les études Philosophiques 3: 315-329

Bradley, KR (1987) Slaves and Masters in the Roman Empire. A Study in Social Control (Oxford)

Brélaz, C (2005) La sécurité publique en Asie Mineure sous le Principat (I - III s. ap. J.C.). Institutions municipales et institutions impériales dans l'Orient romain (Bâle)

Cavaggioni, F (1998) L. Apuleio Saturnino tribunus plebis seditiosus (Venezia)

Chilver, GEF \& Townend, GB (1985) A Historical Commentary on Tacitus' Histories IV and V (Oxford)

Doblhofer, G (1990) Die Popularen der Jahre 111-99 vor Christus (Wien-Köln)

Dumézil, G (1952) “La bataille de Sentinum. Remarques sur la fabrication de l'histoire romaine" Annales 7: 145-154

Fritz, K von (1957) "Tacitus, Agricola, Domitian and the problem of the Principate" Classical Philology 52: 73-97

Gelzer, M (1963) "Nasicas Widerspruch gegen die Zerstörung Karthagos" Kleine Schriften 2 (Wiesbaden): 39-72

Gendre, M \& Loutsch, C (2001) “C. Duilius et M. Atilius Regulus” in Coudry, M \& Späth, T (eds) L'invention des grands hommes de la Rome antique (Paris): 131-172

Hennig, D (1975) L. Aelius Seianus. Untersuchungen zur Regierung des Tiberius (München)

Levick, B (1993) Claudius (Yale)

Levick, B (1999) Vespasian (London)

Liebs, D (2010) Hofjuristen der römischen Kaiser bis Justinian (München)

Lintott, A (1999) Violence in Republican Rome (Oxford)

Meinecke, F (1998) Machiavellism (New Brunswick)

Michel, JH (1980) “L’extradition du général en droit romain” Latomus 39: 675-693 


\section{LA RAISON D'ETAT DANS LA ROME ANTIQUE}

Mix, ER (1970) Marcus Atilius Regulus: Exemplum Historicum (The Hague \& Paris)

Morgan, G (2006) 69 A.D. The Year of Four Emperors (Oxford)

Nemo, P (1998) Histoire des idées politiques dans l'Antiquité et au Moyen-Âge (Paris)

Nippel, W (1995) Public Order in Ancient Rome (Cambridge)

Nörr, D (1983) "C. Cassius Longinus: Der Jurist als Rhetor (Bemerkungen zu Tacitus, Ann. 14.42-

45)" in Heinen, H (ed) Althistorische Studien. Festschrift H. Bengtson (Wiesbaden): 187-222

Oakley, SP (2005) A Commentary on Livy. Books VI-X (Oxford)

Plaumann, G (1913) "Das sogenannte Senatusconsultum ultimum, die Quasidiktatur der späteren Republik" Klio 13: 321-386

Syme, R (1958) Tacitus (Oxford)

Syme, R (1982) Salluste (Besançon)

Syme, R (1989) The Augustan Aristocracy (Oxford)

Ternes, CM (1990) “Le discours de Cerialis aux Trévires et aux Lingons (Tac., Hist. 4, 73sq): une charte de la romanisation?" REL 68: 112-122

Tuori, K (2016) The Emperor of Law. The Emergence of Roman Imperial Adjudication (Oxford)

Ungern-Sternberg, J von (1970) Untersuchungen zum spätrepublikanischen Notstandsrecht. Senatus consultum ultimum und hostis-Erklärung (München)

Wellesley, K (2000) The Year of the Four Emperors (London \& New York) 